The International

Journal of

Integrated

Engineering

\title{
Five-Class SSVEP Response Detection using Common- Spatial Pattern (CSP)-SVM Approach
}

\author{
Mamunur Rashid $^{1 *}$, Norizam Sulaiman'1, Mahfuzah Mustafa ${ }^{1}$, Bifta Sama \\ Bari $^{1}$, Md Jahid Hasan ${ }^{2}$
}

${ }^{1}$ Faculty of Electrical and Electronics Engineering Technology,

Universiti Malaysia Pahang, Pekan, Pahang, 26600, MALAYSIA

${ }^{2}$ Faculty of Manufacturing \& Mechatronic Engineering Technology,

Universiti Malaysia Pahang, Pekan, Pahang, 26600, MALAYSIA

*Corresponding Author

DOI: https://doi.org/10.30880/ijie.2020.12.06.019

Received 27 March 2020; Accepted 15 July 2020; Available online 30 July 2020

\begin{abstract}
Brain-computer interface (BCI) technologies significantly facilitate the interaction between physically impaired people and their surroundings. In electroencephalography (EEG) based BCIs, a variety of physiological responses including P300, motor imagery, movement-related potential, steady-state visual evoked potential (SSVEP) and slow cortical potential have been utilized. Because of the superior signal-to-noise ratio (SNR) together with quicker information transfer rate (ITR), the intentness of SSVEP-based BCIs is progressing significantly. This paper represents the feature extraction and classification frameworks to detect five classes EEG-SSVEP responses. The common-spatial pattern (CSP) has been employed to extract the features from SSVEP responses and these features have been classified through the support vector machine (SVM). The proposed architecture has achieved the highest classification accuracy of $88.3 \%$. The experimental result proves that the proposed architecture could be utilized for the detection of SSVEP responses to develop any BCI applications.
\end{abstract}

Keywords: EEG, BCI, SSVEP, CSP, SVM, Machine Learning

\section{Introduction}

Individual's activities and behavior are entirely controlled through brain waves [1]. The signals from the brain are conveyed to each organ of the body through the nervous system. Due to neuromuscular disorders including amyotrophic lateral sclerosis (ALS) and locked-in syndrome, the individuals' motor functions are lost [2]. In those instances, the individual cannot communicate with others utilizing any mode of intelligence or expression [3]. To come up with a clarification, researchers are attempting to discover a wide range of assistive appliances. The idea of BCI is extensively studying by researchers among those assistive appliances. In every BCI technology, the particular cognitive task has been interpreted into device command which can be utilized in the handling of assistive appliances [4][3]. Brain-operated wheelchair, domestic equipment controlling, robotic arm commanding, spelling technology, workload recognition, and authentication detection system are the widely adopted BCI applications [5] [6].

Non-invasive and invasive approaches have been employed to collect brain activity from the physically impaired people. Two invasive procedures including, intracortical neuron recording and electrocorticography (ECoG) have been adopted in the BCI study [7]. Due to the risks of health hazers, the invasive modalities are comparatively less 
used by the researchers. The EEG, MEG, NIRS, and fMRI are extensively adopted non-invasive architectures [8] [7]. The cognitive tasks are extracted in such a fashion that the thoughts of a physically impaired individual can interpret and regulate the BCI based assistive devices [9]. Those cognitive signals can be considered as the EEG control signals. The extensively adopted cognitive control signals in the BCI community are the SSVEP, P300, motor imagery and SCP [10] [7]. Because of the superior SNR together with quicker ITR, the intentness of SSVEP-based BCIs is progressing significantly [11].

Every BCI system essentially consists of five components which are the brain activity capturing, noise removal, feature extraction and classification; finally, device command. All the components of BCI system has been illustrated in Fig. 1. Among those components, feature extraction and classification perform an essential character to boost up the inclusive accuracy of any BCI technology. A large number of feature extraction and classification approaches have employed to recognize the SSVEP responses over the last few years.

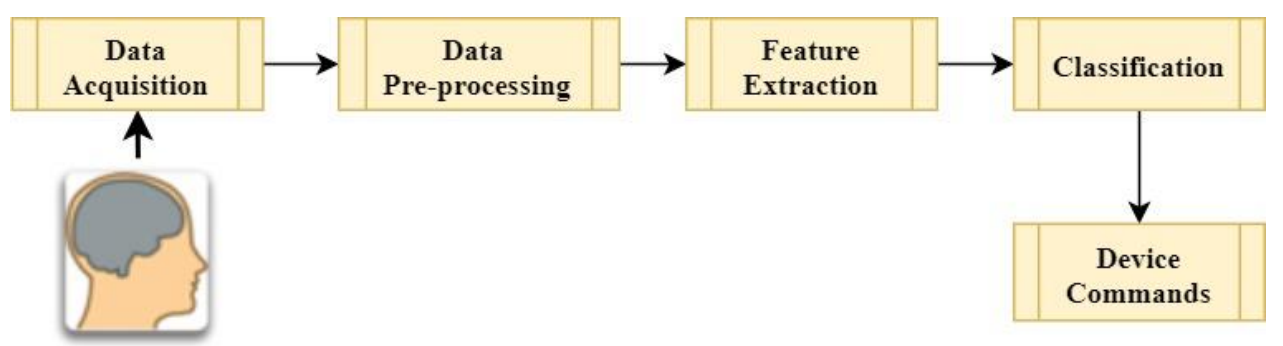

Fig. 1 - Components of the BCI system

Regarding the time-domain based EEG features, the aggregation of EEG samples weight has been utilized in articles [12] [13]. The spectral analysis approach has been investigated to extract the feature in the form of frequencydomain from the SSVEP responses. Particularly, the periodogram architecture has been utilized to assess the spectral properties of the SSVEP based EEG in [14] [15] [16]. Other spectral based features-extraction methods including fast Fourier transform (FFT) [17] and Hilbert transform (HT) [18] have been employed in the purpose of targeted frequencies recognition from the single electrode EEG amplifier. An innovative method has been projected in [19], where the phase and frequency have been combined to add more target frequencies A power spectral density (PSD)based approach, known as the Welch method, has been employed in reference [20]. Here, authors have also investigated the time-frequency based feature extraction approaches known as spectrogram.

Finally, several machine learning-based classification algorithms have been utilized for recognizing specific frequency of SSVEP responses. In reference to [20], machine learning-based classifiers including SVM, extreme learning machines (ELM) and linear discriminant analysis (LDA) have been utilized. The LDA as well as SVM are the extensively adopted classifiers to categorize SSVEP responses and in considerable number of studies including [20] [13] [21], have been utilized those classifiers. Besides, the adaptive network based fuzzy inference scheme has been utilized in [12]. Authors in [21], has been developed neural networks (NN) to categorize the SSVEP responses. In reference to [22], a statistic test has been employed to make the choice, while in [15] a group of precepts has been implemented on spectral features.

This study exposes that the common-spatial pattern (CSP) has been employed for feature extraction from SSVEP responses and these features have been classified through the SVM. The present article has been prepared in subsequent segments i.e. section 2 presents methodology whereas results and discussion has been illustrated in section 3 ; lastly, section 4 concludes with the conclusion.

\section{Methodology}

Generally, a BCI system consists of five essential components which are data capturing, data pre-processing, feature extraction, classification as well as device command. Compared to other three components, feature extraction and classification architecture are the most vital steps which may significantly contribute to increase the overall BCI performance. Fig.2 illustrates the complete flow chart of this study. This study has been conducted with the publicly available online dataset. The dataset consists of five classes EEG-SSVEP response. The first step of this study is the data pre-processing where the entire dataset has been filtered. A $5^{\text {th }}$ order Butterworth bandpass filter has been designed to filter the data. The frequency range of this filter was $4 \mathrm{~Hz}$ to $30 \mathrm{~Hz}$. The filtered data has been utilized to extract the feature. In this study, the feature has been extracted through the CSP. After extracting the feature, the entire dataset has been split into two groups: namely, training and testing dataset. The training and testing ratio were 75:25. The training dataset has been utilized to train the model. An SVM architecture has been utilized as the training model. Once the model is trained, the confusion matrix, classification accuracy, true positive and false negative rate are utilized to evaluate the performance of the trained model. The optimum performance has been achieved by randomly changing the classifier parameters. After obtaining optimum accuracy, the testing dataset are used to test the trained model. The overall performance of this study has been assessed with the testing performance. 
The MATLAB environment (MATLAB 2019b) has been utilized to conduct the complete SSVEP data analysis phase. For classification purposes, Classifier Learner Apps (a built-in toolbox of MATLAB) has been employed to classify the SSVEP features using the machine learning algorithms.

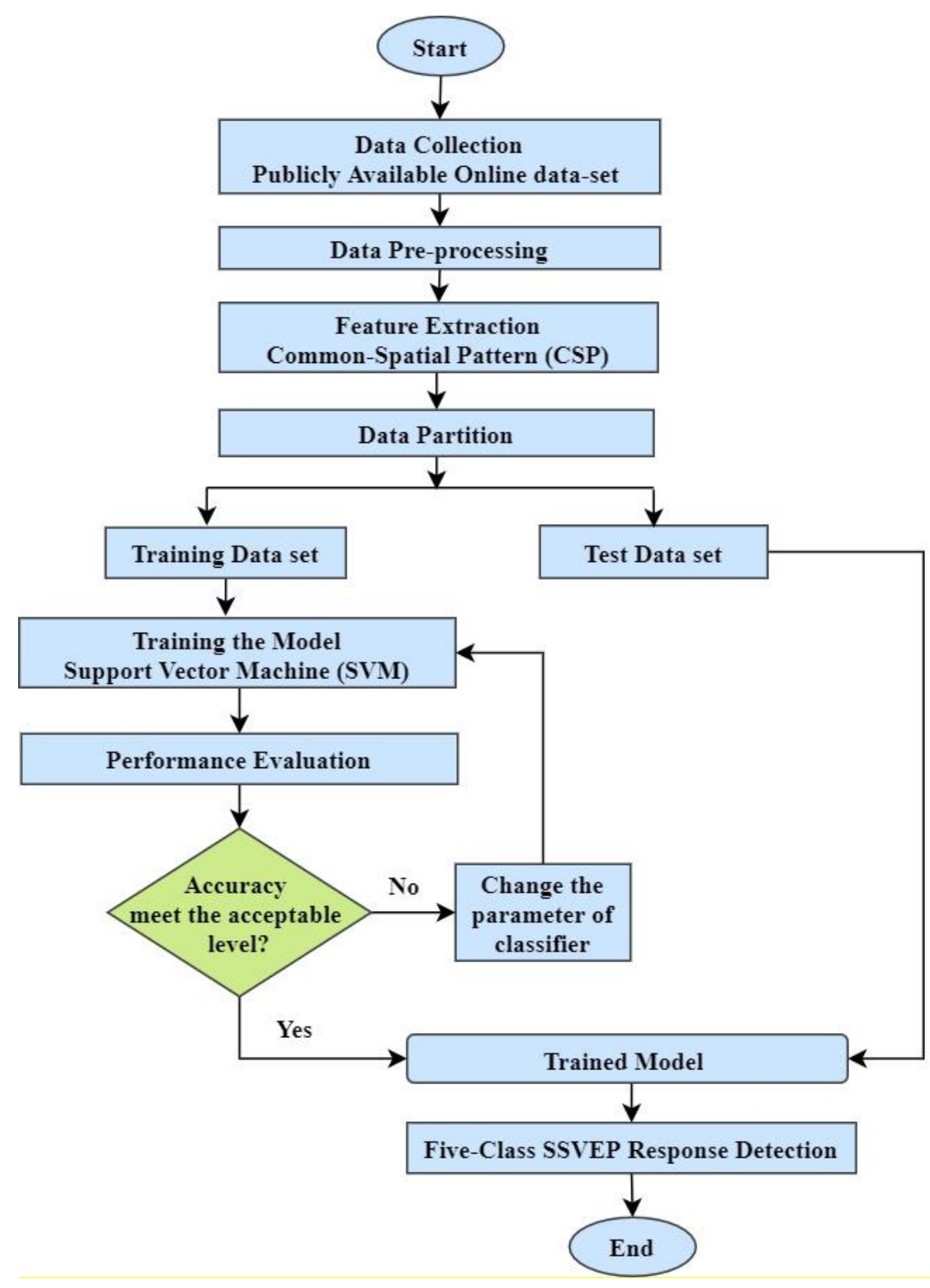

Fig. 2 - Complete methodology of the experiment

\subsection{Detailed Description of SSVEP Dataset}

In this study, the five-classes EEG-SSVEP data has been utilized. The dataset has been taken from MAMEMDataset EEG SSVEP Dataset III [23]. Eleven subjects contributed to the data acquisition process. The number of males were 8 and the number of females were 3 among the subjects. The ages vary from 25 to 39 years. The individuals were normal in physical condition and did not have any mental or neuro-muscular disorders. Subjects can be categorized based on the hair length and thickness into 3 categories, short hair, regular hair, and thick hair, with 3 belonging to the first category, 6 to the second and the remaining 4 to the third. Table 1 summarizes the demographic information about the participating subjects. 
Table 1 - Descriptions of the participating subjects [23]

\begin{tabular}{lcccc}
\hline Subject & Age & Gender & Hair Type & Handedness \\
\hline Subject-1 & 24 & Male & Regular & Right-handed \\
\hline Subject-2 & 37 & Male & Regular & Right-handed \\
\hline Subject-3 & 39 & Male & Thick & Right-handed \\
\hline Subject-4 & 31 & Male & Short & Right-handed \\
\hline Subject-5 & 27 & Female & Thick & Left-handed \\
\hline Subject-6 & 28 & Female & Thick & Right-handed \\
\hline Subject-7 & 26 & Male & Regular & Right-handed \\
\hline Subject-8 & 31 & Female & Thick & Right-handed \\
\hline Subject-9 & 29 & Male & Short & Right-handed \\
\hline Subject-10 & 37 & Male & Regular & Right-handed \\
\hline Subject-11 & 25 & Male & Regular & Right-handed \\
\hline
\end{tabular}

The visual stimuli have been shown upon a 22" LCD monitor by fixing the pixel resolution at $1680 \times 1080$ and refresh rate at $60 \mathrm{~Hz}$. The EEG data were recorded in better grade by14 wireless electrodes where the sampling rate was $128 \mathrm{~Hz}$. Fig. 3(a) illustrates the electrode positioning system of the the Emotiv Epoc.

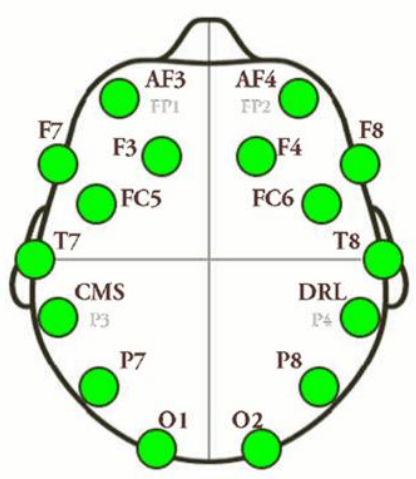

(a)

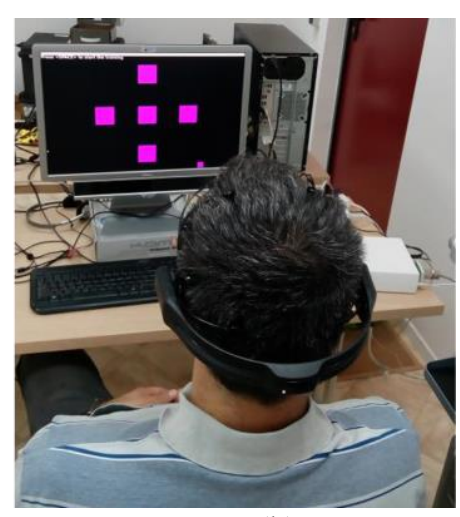

(b)

Fig. 3 - (a) Electrode positioning system; (b) Experimental setup [23]

The experimental stimuli consist of five violet boxes in number which have been flickered simultaneously in five dissimilar frequency $(6.66,7.50,8.57,10.00$ and $12.00 \mathrm{~Hz})$. Each of those flickering boxes had their individual frequency where they were showed for five seconds each simultaneously, denoted as trial, then a gap of five seconds without any visual stimulation before the further appearance of the flickering boxes. Before the occurrence of stimulation, the subjects had to focus on a box marked by a yellow mark (Fig. 3(b)). In the whole experiment, the background was black. The experiment was started with giving the subjects a period of 100 seconds for adaptation.

Each subject conducted five identical sessions followed the adaptation period. Each session includes 25 trials and is divided into two parts by a 30-second resting period. The first part includes 12 trials, whereas the second is 13 . The target in each trial is being selected in a random way in order to avoid habituation. The total number of trials of this dataset was 1375 .

\subsection{Common-Spatial Pattern (CSP)}

Among the most widespread feature extraction techniques in EEG based BCIs, CSP is well-known approach which is employed in this study. As the spatial filters are provided by CSP technique, it enlarges the variance of one class whereas the variance of the other class is minimized simultaneously [24]. The differences between two classes can be done by standard CSP, an extension in the original method in [25] for multi-class paradigm. The one vs. rest approach is partitioning the k-class problem into a set of $\mathrm{k}$ binary classes while differentiating each class against the remaining classes. For an EEG trial, the normalized spatial covariance matrix is defined by the following equation: 


$$
C_{\text {class }}=\frac{A A^{T}}{\operatorname{trace}\left(A A^{T}\right)}
$$

Where, A symbolizes a trial wich is [Ch $\mathrm{x} \mathrm{S}]$ matrix (Ch is the electrodes number and $\mathrm{S}$ is the samples number) and class is the types of MI activity in the dataset. The trace $\operatorname{trace}\left(A A^{T}\right){ }^{\operatorname{trace}\left(A A^{T}\right)}$ is the summation of diagonal components of matrix $A A^{T}$. The mean normalized covariance of each group is computed by performing mean over each class containing all trials. The following equation shows the compound spatial covariance:

$$
C_{r}=\sum_{\text {class }=1}^{m} \overline{C_{\text {class }}}
$$

Where, $\mathrm{m}$ is the whole quantity of class in the MI data. The covariance matrices of disjoint trials $C_{r}^{\prime}$ to the all groups will be

$$
C_{r}^{\prime}=\sum_{k=c l a s s} C_{k}
$$

Where, $\mathrm{k}=\{1,2,3 \ldots \ldots \mathrm{m}\}$. The composite covariance can be factorized as

$$
C_{r}=E_{0} \Delta E_{0}^{T}
$$

where, $\Delta$ denotes the diagonal matrix of eigenvalues of order $\mathrm{N} \times \mathrm{N}$ and $E_{0}$ denotes the $\mathrm{N} \times \mathrm{N}$ matrix of Eigenvectors. Then, $R_{W}$ denotes a whitening transformation that balances the variances in eigenspace, has been computed by the following equation:

$$
R_{W}=\Delta^{-\frac{1}{2}} E_{0}^{T}
$$

The whitening matrix $R_{W}$ has been utilized to transform the mean covariance matrices as

$$
Q_{\text {class }}=R_{W} \bar{C}_{\text {class }} R_{W}^{T} \text { and } Q_{k}=R_{W} C_{r}^{\prime} R_{W}^{T}
$$

Then, $Q_{\text {class }}$ and $Q_{k}$ exchange common eigenvectors. The sum of the corresponding eigenvalues for the two matrices must be identical to 1 ,

$$
\begin{gathered}
Q_{\text {class }}=P_{\text {class }} \Delta_{\text {class }} P_{\text {class }}^{T} \text { and } Q_{k}=P_{\text {class }} \Delta_{r}^{\prime} P_{\text {class }}^{T} \\
\Delta_{\text {class }}+\Delta_{r}^{\prime}=I
\end{gathered}
$$

Where, $I$ signify identity matrix. Finally, a projection matrix can be computed by

$$
L=\left(P_{\text {class }}^{T} R_{W}\right)^{T}
$$

Where, the columns $L^{-1}$ is the CSP and might be represented as time-invariant EEG source distribution vectors. By having the projection matrix, the decomposition of a trial $S$ has been computed by the following Equation:

$$
X=S L
$$

The variances of the initial as well as final rows of $\mathrm{X}$ are appropriate classification features because one is a constant value as the sum of the corresponding eigenvalues. Here, we have utilized the variances of the first and last rows as features whereas the variance has been computed as follows:

$$
V_{r}=\frac{\sum\left(X_{R}-\overline{X_{R}}\right)^{2}}{Z-1}
$$

Where, $X_{R}$ denotes a row of $\mathrm{X} ; \mathrm{Z}$ is defined as the length of this row.

\subsection{Support Vector Machine (SVM)}

The SVM classifier generates a hyperplane to isolate the feature vectors into individual classes as illustrated in Fig. 4. The SVM, in oppose to LDA, selects a hyperplane to maximize the margins which sets the distance between the nearest training samples as well as the hyperplanes [26] to the largest. Linear SVM is a classification that empowers classification using linear decision boundaries. To a significant number of synchronous BCI problems [27] this classifier has been applied successfully. Nevertheless, using the "kernel trick" nonlinear decision boundaries is efficient with a small increment of the classifier's complexity. The common kernel used in BCI research is "kernel 
trick". The corresponding SVM is called Gaussian SVM or RBF SVM. To obtain better results the RBF SVM is proved to be robust in BCI applications [27], [28]. In BCI applications, the SVM is commonly popular as the simpler algorithm. Additionally, it is robust in higher dimensional datasets that does not require large training datasets for better results even when the feature vectors are high in dimension [29]. Moreover, the execution speed is not hindered by these favorable circumstances during the integration of the real-time BCIs [30]. Nonetheless, to increase the classification accuracy of SVM structure, an important role is played by several sorts of kernel function along with the regularization parameter C. The most used kernel functions of SVM are Gaussian radial basis function (RBF), polynomial, sigmoid and linear. The Gaussian RBF kernel function has been used in this research. At last, the few hyperparameters SVM possess which requires identification by hand are the regularization parameter $\mathrm{C}$ and the $\mathrm{RBF}$

width $\sigma$ when utilizing kernel 2 .

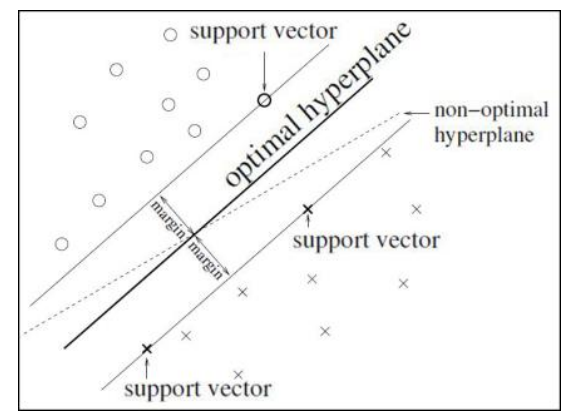

Fig. 4 - SVM find the optimal hyperplane for generalization [31]

In the present study, the Gaussian or RBF kernel has been utilized which is given by the Equation (12)

$$
K(\mathrm{x}, \mathrm{y})=\exp \left(\frac{-\|x-y\|^{2}}{{ }^{2} \sigma^{2}}\right)
$$

Here, $K(\mathrm{x}, \mathrm{y})$ is the kernel function and $\sigma$ is the RBF width.

\section{Results and Discussion}

Before performing the training session of the classifier, the training dataset has been marked with labels. In this study, 5 different flickering frequencies namely 6.66, 7.50, 8.57, 10.00 and $12.00 \mathrm{~Hz}$ have been utilized. During labeling, 6.66, 10.00, 8.57, 12.00 and $7.50 \mathrm{~Hz}$ has been denoted by $1,2,3,4$ and 5 respectively. The experimental datasets consist of total 1375 trials where class-1, class- 2 and class- 4 have 275 trials in each class. Class- 3 and class5 consists of 330 and 220 trials respectively. After splitting the dataset into training and testing phase, the number of testing trials for class-1, class-2, class-3, class-4 and class-5 are 69, 68, 82, 69 and 55 respectively. These trials have been utilized to test the trained model. The confusion matrix (see Fig 5 a), true positive rate (TPR) as well as falsenegative rate (FNR) (see Fig 5 b) are represented by Fig. 5. It could be interpreted from the confusion matrix that the 303 observations (out of 343 testing observations) have been identified precisely by the trained model. Hence, the classification accuracy is $88.3 \%$. The reasons behind the lower classification accuracy are the flickering frequencies 10.00 and $8.57 \mathrm{~Hz}$ that is class- 2 and class-3. In class- 2 and class-3, the TPR are $81 \%$ and $82 \%$ respectively. Whereas, the FNR for class- 2 and class- 3 are $19 \%$ and $18 \%$ respectively which are very high. In class-1, class- 4 and class- 5 , the TPR are $94 \%, 90 \%$ and $98 \%$ respectively which are very encouraging. 


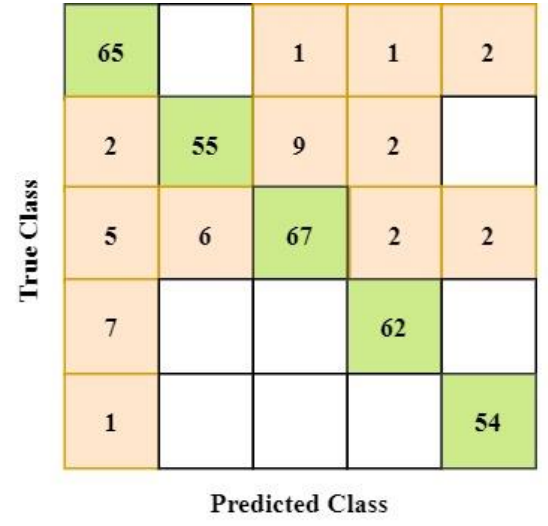

(a)
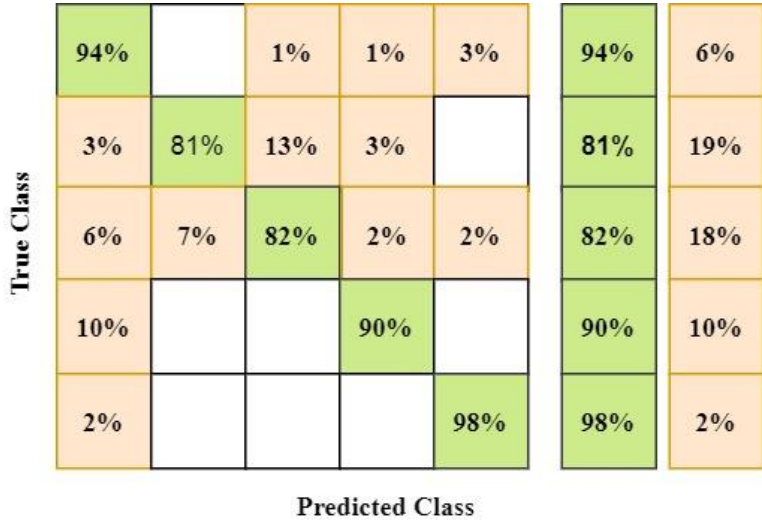

(b)

Fig. 5 - (a) Confusion matrix; (b) True positive and false negative rate

The performance of the classifier can also be assessed through the area under curve (AUC) - receiver operating characteristics (ROC) in machine learning. The ROC is the probability curve while the AUC describes the level of separability. This metric demonstrates the model's competence in distinguishing the groups. The value of AUC ranges from 0 to 1 . The greater value of AUC signifies that the model could recognize the groups precisely. Fig. 6 illustrates the classifier performance with respect to the AUC-ROC. The AUC of class-1, class-4 and class-5 are 0.98, 0.99 and 1.00 respectively which indicate the excellent classifier performance. However, the AUC of class- 2 and class- 3 are 0.96 which indicates the lower classifier performance as compared to the other classes.
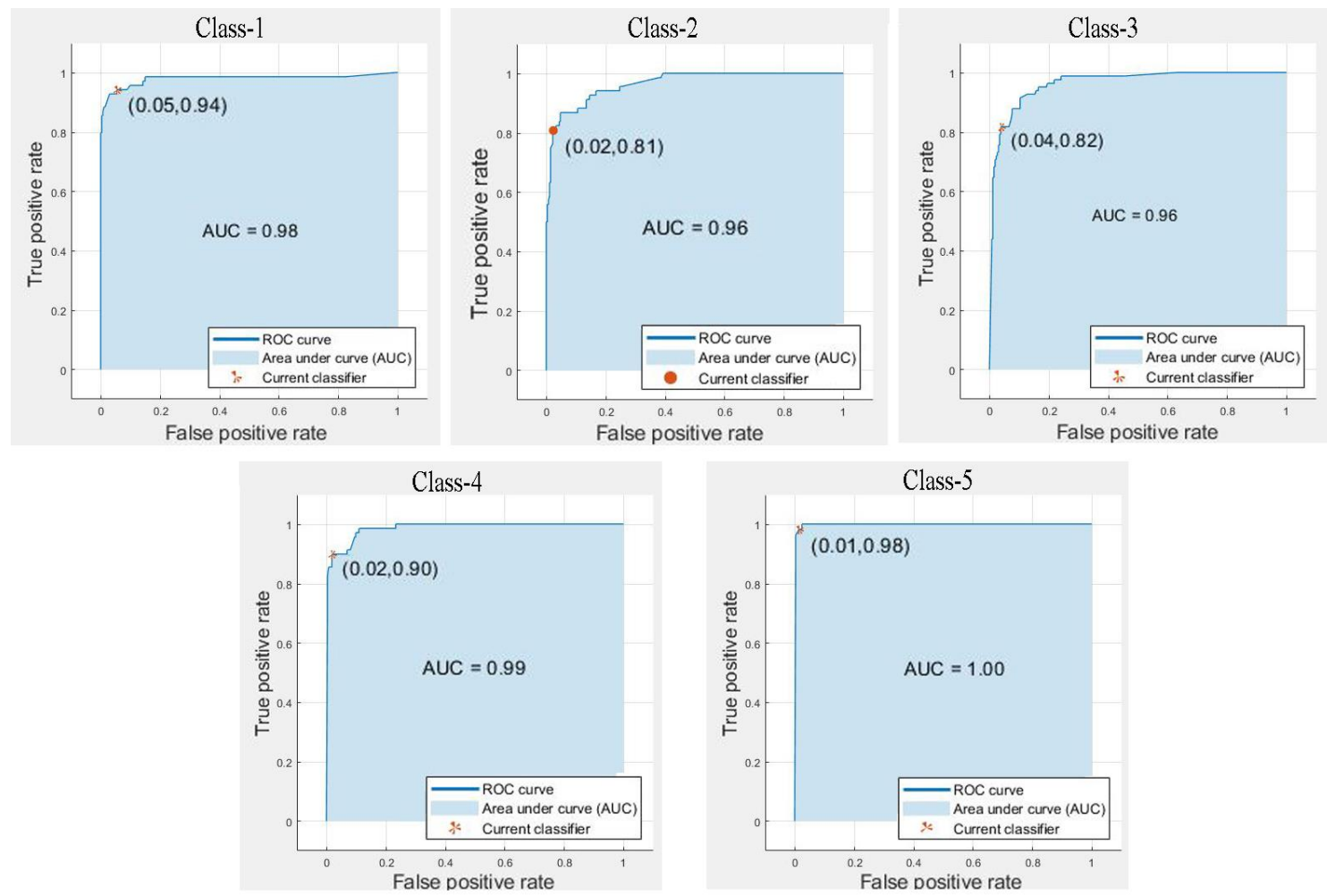

Fig. 6 - AUC curve- ROC curve

\section{Conclusion}

In this study, five-classes EEG-SSVEP responses have been recognized. In order to do this, the features from SSVEP responses have been extracted through the CSP and the extracted feature has been classified by the extensively employed SVM based machine learning algorithm. To evaluate the classifier performance, some evaluation metrics including AUC-ROC, accuracy, confusion matrix, FNR and TPR have been computed. The obtained accuracy is very encouraging. However, there are some issues that need to be overcome. The conducted experiment utilized publicly available online dataset which has been captured from the healthy subjects. However, the fundamental motivation 
behind the BCI technology is to help the physically impaired patients, the experimental data must be collected from those individuals who are the targeted consumers. From the findings of this study, it clear that the classification accuracy is not still sufficient in respect of the absolute BCIs. Therefore, the accuracy should be improved. In order to make real-life BCI applications, the classifier outcomes need to be converted into device commands and in the meantime, the entire operation should be performed in real-time.

\section{Acknowledgement}

The author would like to acknowledge the magnificent supports from the Faculty of Electrical \& Electronics Engineering Technology and Universiti Malaysia Pahang to provide fundamental research grant scheme to support this research, FRGS/1/2018/TK04/UMP/02/3 (RDU 190109).

\section{References}

[1] Lin, J.-S., and Shieh, C.-H. (2014). An Ssvep-Based Bci System and its Applications. Int. J. Adv. Comput. Sci. Appl. 5.

[2] Mistry, K. S., Pelayo, P., Anil, D. G., and George, K. (2018). An SSVEP Based Brain Computer Interface System to Control Electric Wheelchairs. in 2018 IEEE International Instrumentation and Measurement Technology Conference (I2MTC) (Houston, TX: IEEE), 1-6

[3] Rashid, M., Sulaiman, N., Mustafa, M., Sama, B., and Golam, B. (2020). Wink based facial expression classification using machine learning approach. SN Appl. Sci. 2

[4] Rahman, K. A. A., Ibrahim, B. S. K. K., Jamil, M. M. A., and Leman, A. M. (2012). The Development of control system via Brain Computer Interface (BCI)-Functional Electrical Stimulation (FES) for paraplegic subject. Int. J. Integr. Eng. 4, 59-64

[5] Science, C., and Info, A. (2019). Performance of channel selection used for multi-class EEG signal classification of motor imagery. Indones. J. Electr. Eng. Comput. Sci. 15, 1305-1312

[6] Rashid, M., Sulaiman, N., Mustafa, M., Khatun, S., and Bari, B. S. (2019). "The Classification of EEG Signal Using Different Machine Learning Techniques for BCI Application,” in Robot Intelligence Technology and Applications. RiTA 2018. Communications in Computer and Information Science, vol 1015, ed. S.-M. L. Jong-Hwan, KimHyung Myung (Springer Singapore), 207-221

[7] Rashid, M., Islam, M., Sulaiman, N., Sama, B., Ripon, B., Saha, K., et al. (2020). Electrocorticography based motor imagery movements classification using long short - term memory (LSTM) based on deep learning approach. SN Appl. Sci. 2

[8] Dania, A., Rahman, A., and Hussin, H. (2019). Detection of attention and meditation state-based brainwave system to control prosthetic arm. Indones. J. Electr. Eng. Comput. Sci. 13, 794-800

[9] Nicolas-Alonso, L. F., and Gomez-Gil, J. (2012). Brain computer interfaces, a review. Sensors

[10] Wen, T. Y., and Aris, S. A. M. (2019). Electroencephalogram (EEG) stress analysis on alpha/beta ratio and theta/beta ratio. Indones. J. Electr. Eng. Comput. Sci. 17, 175-182

[11] Trigui, O., Zouch, W., Slima, M. Ben, and Messaoud, M. Ben (2018). Bispectral analysis-based approach for steady-state visual evoked potentials detection. Multimed. Tools Appl. 78, 12865-12882

[12] Martinez, P., Bakardjian, H., and Cichocki, A. (2007). Fully online multicommand brain-computer interface with visual neurofeedback using SSVEP paradigm. Comput. Intell. Neurosci. 2007

[13] Guger, C., Allison, B. Z., Großwindhager, B., Prückl, R., Hintermüller, C., Kapeller, C., et al. (2012). How Many People Could Use an SSVEP BCI? Front. Neurosci. 6

[14] Wang, Y., Wang, R., Gao, X., Hong, B., and Gao, S. (2006). A practical VEP-based brain-computer interface. in IEEE Transactions on Neural Systems and Rehabilitation Engineering, 234-239

[15] Vilic, A., Kjaer, T. W., Thomsen, C. E., Puthusserypady, S., and Sorensen, H. B. D. (2013). DTU BCI speller: An SSVEP-based spelling system with dictionary support. in Proceedings of the Annual International Conference of the IEEE Engineering in Medicine and Biology Society, EMBS, 2212-2215

[16] Müller-Putz, G. R., Scherer, R., Brauneis, C., and Pfurtscheller, G. (2005). Steady-state visual evoked potential (SSVEP)-based communication: Impact of harmonic frequency components. J. Neural Eng. 2, 123-130

[17] Ortner, R., Allison, B. Z., Korisek, G., Gaggl, H., and Pfurtscheller, G. (2011). An SSVEP BCI to control a hand orthosis for persons with tetraplegia. IEEE Trans. Neural Syst. Rehabil. Eng. 19, 1-5

[18] Zhao, L., Yuan, P., Xiao, L., Meng, Q., Hu, D., and Shen, H. (2010). Research on SSVEP feature extraction based on HHT. in Proceedings - 2010 7th International Conference on Fuzzy Systems and Knowledge Discovery, FSKD 2010, 2220-2223 
[19] Jia, C., Gao, X., Hong, B., and Gao, S. (2011). Frequency and phase mixed coding in SSVEP-based brain Computer interface. IEEE Trans. Biomed. Eng. 58, 200-206

[20] Carvalho, S. N., Costa, T. B. S., Uribe, L. F. S., Soriano, D. C., Yared, G. F. G., Coradine, L. C., et al. (2015). Comparative analysis of strategies for feature extraction and classification in SSVEP BCIs. Biomed. Signal Process. Control

[21] Singla, R. (2014). Comparison of SSVEP Signal Classification Techniques Using SVM and ANN Models for BCI Applications. Int. J. Inf. Electron. Eng. 4, 6-10

[22] Friman, O., Volosyak, I., and Gräser, A. (2007). Multiple channel detection of steady-state visual evoked potentials for brain-computer interfaces. IEEE Trans. Biomed. Eng. 54, 742-750

[23] Nikolopoulos, S. (2016). MAMEM EEG SSVEP Dataset III (14 channels, 11 subjects, 5 frequencies presented simultaneously). figshare, Dataset

[24] Ramoser, H., Müller-Gerking, J., and Pfurtscheller, G. (2000). Optimal spatial filtering of single trial EEG during imagined hand movement. IEEE Trans. Rehabil. Eng. 8, 441-446

[25] Dornhege, G., Blankertz, B., Krauledat, M., Losch, F., Curio, G., and Müller, K. R. (2006). Combined optimization of spatial and temporal filters for improving brain-computer interfacing. IEEE Trans. Biomed. Eng. 53, 2274-2281

[26] Burges, C. J. C. (1998). A Tutorial on Support Vector Machines for Pattern Recognition. Data Min. Knowl. Discov. 2, 121-167

[27] Garrett, D., Peterson, D. A., Anderson, C. W., and Thaut, M. H. (2003). Comparison of linear, nonlinear, and feature selection methods for eeg signal classification. IEEE Trans. Neural Syst. Rehabil. Eng. 11, 141-144

[28] Rakotomamonjy, A., Guigue, V., Mallet, G., and Alvarado, V. (2005). "Ensemble of SVMs for Improving Brain Computer Interface P300 Speller Performances," in (Springer, Berlin, Heidelberg), 45-50

[29] Kaper, M., Meinicke, P., Grossekathoefer, U., Lingner, T., and Ritter, H. (2004). BCI Competition $2003-$ Data Set IIb: Support Vector Machines for the P300 Speller Paradigm. IEEE Trans. Biomed. Eng. 51, 10731076

[30] Thulasidas, M., Guan, C., and Wu, J. (2006). Robust Classification of EEG Signal for Brain-Computer Interface. IEEE Trans. Neural Syst. Rehabil. Eng. 14, 24-29

[31] Lotte, F., Congedo, M., Lécuyer, A., Lamarche, F., and Arnaldi, B. (2007). A review of classification algorithms for EEG-based brain-computer interfaces. J. Neural Eng. 4, R1-R13 